\title{
Methylphenidate enhances implicit learning in healthy adults
}

\author{
Corinna Klinge ${ }^{1,2}$, Claire Shuttleworth ${ }^{1}$, Pierandrea Muglia ${ }^{3}$, Anna C Nobre ${ }^{4,5}$, \\ Catherine J Harmer ${ }^{1,2 *}$, Susannah E Murphy ${ }^{1,2 *}$ \\ * Joint senior authorship
}

Short title: methylphenidate and implicit cognition

Keywords: methylphenidate, Ritalin, healthy volunteers, cognition, learning, implicit

\section{Affiliations}

${ }^{1}$ Department of Psychiatry, University of Oxford, Oxford, United Kingdom

${ }^{2}$ Oxford Health, NHS Foundation Trust, Oxford, UK

${ }^{3}$ UCB pharma, Brussels, Belgium

${ }^{4}$ Oxford Centre for Human Brain Activity, Department of Psychiatry, University of Oxford, Oxford, United Kingdom

${ }^{5}$ Department of Experimental Psychology, University of Oxford, Oxford, United Kingdom

Corresponding author: Corinna Klinge; corinna.klinge@psych.ox.ac.uk; tel: +44(0)1865 618304; Department of Psychiatry, University of Oxford, Oxford, UK 


\section{ABSTRACT}

Background and Purpose. One limiting factor in the development of pharmacological interventions to enhance cognition is the absence of biomarkers that can be used in healthy volunteers to screen novel compounds. Drug discovery has tended to rely heavily on explicit measures of cognition, but these are typically insensitive to cognition enhancing effects in healthy volunteers. This study investigated whether a novel battery of implicit cognition measures is sensitive to the effects of methylphenidate (Ritalin) in healthy volunteers.

Experimental Approach. 80 healthy volunteers were randomised to receive either a single (10mg) dose of methylphenidate or matched placebo. Participants completed a battery of tasks measuring implicit cognition (location priming, contextual cueing, implicit task switching). The effect of methylphenidate on standard, explicit measures of cognition was also assessed.

Key Results. Methylphenidate enhanced implicit learning on the location priming task and the implicit task-switching task. In line with previous work, we found these effects were greater in male volunteers. There was no evidence for improved learning in any of the explicit measures.

Conclusion and Implications. These results demonstrate that implicit measures of cognition are sensitive to pharmacological interventions in healthy volunteers. As such, implicit cognition measures may be a useful way of screening and tracking cognitive effects of novel agents in experimental medicine studies. 


\section{ABBREVIATIONS}

Acq - Acquisition

ADHD - Attention Deficit Hyperactivity Disorder

BDI - Beck’s Depression Inventory

BMI - Body Mass Index

DA - Dopamine

DSM - Diagnostic and Statistical Manual of Mental Disorders

ED acq - Extra-dimensional Acquisition

ED rev - Extra-dimensional reverse

EEG - Electroencephalography

mg - milligram

NA - noradrenaline

NART - National Adult Reading Test

PANAS - Positive and Negative Affect Schedule

PDQ - Perceived Deficits Questionnaire

PET - Positron Emission Tomography

Rev - reverse 
RT - Reaction time

STAI - State-Trait Anxiety Inventory

THC - Tetrahydrocannabinol

VAS - Visual Analogue Scale

WM - working memory 


\section{INTRODUCTION}

There is increasing awareness of the frequency and severity of cognitive dysfunction associated with psychiatric and neurological disorders (Millan et al., 2012). Pharmacological treatments for the cognitive deficits associated with disorders such as Attention Deficit Hyperactivity Disorder (ADHD) are in widespread and increasing use, however, there remains significant unmet need in disorders such as Parkinson's and Alzheimer's Disease. Indeed, prescriptions of the dopamine and noradrenaline reuptake inhibitor methylphenidate, used primarily in the treatment of ADHD, increased by 176\% in England between 2004 and 2015 (NHS Prescription Cost Analysis England 2004 and 2015).

There is, therefore, intense interest in the discovery and development of drugs that restore and enhance cognitive function. However, currently available 'cognitive enhancers' appear to have only relatively modest effects (Husain and Mehta, 2011) and there is a pressing need to develop more effective pharmacological interventions to enhance cognition. An important limiting factor in the development of novel cognition enhancing drugs is the lack of reliable and sensitive biomarkers that can be used to test the effect of pharmacological agents early in development. Animal models have limited predictive validity and there has been a disappointing failure to predict the efficacy of pro-cognitive drugs in humans from drug discovery studies in rodents (Millan et al., 2012). Experimental medicine approaches using healthy volunteer models are an 
alternative way to model the cognitive effects of pharmacological treatments in patients and may represent a useful approach for screening and understanding the mechanisms of candidate agents early in development (Harmer et al., 2011).

Acute administration of methylphenidate (Ritalin) has been reported to improve cognition across a number of different domains in healthy volunteers, including inhibitory control (Vaidya et al., 1998), episodic memory (Linssen et al., 2012), working memory (Agay et al., 2014; Cooper et al., 2005; Elliott et al., 1997; Linssen et al., 2014; Mehta et al., 2000), shifts of attention between stimulus dimensions (Rogers et al., 1999) and sustained attention (Agay et al., 2014). A recent meta-analysis, which combined studies looking at the effects of methylphenidate and amphetamine, concluded that these stimulants have a small enhancing effect on inhibitory control, working memory and short term episodic memory, and a medium-sized enhancing effect on delayed episodic memory in healthy volunteers (Ilieva et al., 2015).

However, the reported effects of methylphenidate and related stimulant drugs on cognition in healthy volunteers are small (Hall and Lucke, 2010; Repantis et al., 2010), highly variable (Linssen et al., 2014; van der Schaaf et al., 2013) and sometimes contradictory (Advokat, 2010; Elliott et al., 1997). Many studies have found these effects to be dependent on baseline cognitive ability, with only participants with relatively low baseline performance levels showing drug-related improvements (Agay et 
al., 2014; del Campo et al., 2013; Mehta et al., 2000). Further, a number of studies have only found effects of methylphenidate on cognition at the most difficult levels on cognitive tasks (Mehta et al., 2000; Vaidya et al., 1998). Such findings may be driven by a lack of sensitivity in the measures used to assess drug-induced cognitive effects in healthy volunteers. Healthy volunteer models are often limited by ceiling effects in performance and by the use of compensatory strategies by high functioning participants, thus limiting the detection of pharmacologically induced changes.

One potentially useful approach to increase the sensitivity of paradigms to drug-related effects is to employ more implicit, automatic measures of cognition. These are designed to assess information processing that is not readily accessible to conscious awareness and that cannot easily be controlled, but that nonetheless influence task performance. While many studies have measured the effects of cognition enhancing drugs on explicit cognitive processes, relatively little is known about their effects on implicit cognition. Given that implicit measures are less vulnerable to ceiling and compensatory strategy effects, they may be relatively more discriminative and sensitive to pharmacological manipulation (Harmer et al., 2011; Pringle et al., 2013). In support of this idea, our preliminary work in this area showed that the wake-promoting agent modafinil improved learning in an implicit attention switching task even in the absence of effects on more explicit measures (Pringle et al., 2013). 
The aim of the current study was therefore to investigate the effect of a low dose of methylphenidate on measures of implicit learning, memory and attention in healthy volunteers. Overall, dopamine-dependent (fronto)-striatal circuits have been shown to play a pivotal role in implicit measures, in healthy controls (e.g. Bauer et al., 2012), as well as in patient studies (e.g. Foerde and Shohamy, 2011; van Asselen et al., 2009). We therefore employed a double-blind, placebo-controlled, between subjects design to investigate the effect of a single low dose of methylphenidate on a battery of implicit cognition tasks, which included measures of location priming, contextual cueing and implicit task switching. We also tested the effects of methylphenidate on established measures of explicit cognition that have been previously been shown to be sensitive to higher doses of methylphenidate (Agay et al., 2014; Clatworthy et al., 2009; del Campo et al., 2013; Elliott et al., 1997; Mehta et al., 2000). We predicted that the implicit measures would be more sensitive to the effects of a low dose of methylphenidate than the explicit measures. Interestingly, a recent meta-analysis found preliminary evidence to suggest that the effects of methylphenidate may be larger in male participants (Ilieva et al., 2015); however, this evidence is limited by the tendency in the existing literature to only test the effects of the drug in male participants (Clatworthy et al., 2009; Cooper et al., 2005; del Campo et al., 2013; Elliott et al., 1997; Mehta et al., 2000). Therefore, a secondary aim of the current study was to investigate whether there are gender differences in the effect of methylphenidate on cognition. 


\section{METHODS}

\section{Participants}

80 right-handed participants, aged 18-30 years, were included in this study (40 female, 40 male). Participants were screened for contraindication to methylphenidate administration, including a physical examination, and were not taking any psychoactive medication (other than the contraceptive pill). A urine drug screen was used to screen for cocaine, amphetamines, methamphetamines, THC, opiates and benzodiazepines. All participants were free of current or past axis 1 disorders (assessed with the Structured Clinical Interview for DSM-IV, SCID-I/NP Non-patient Edition; First et al., 2002). Participants were fluent in English and had a Body Mass Index (BMI) of between 18 and 30. Exclusion criteria included: general anaesthetic within the last 6 months; previous head injury; high caffeine consumption (more than 6 cups/day); smoking more than 7 cigarettes/day; uncorrected problems with hearing or vision. The study was approved by the local ethics committee (University of Oxford Central University Research Ethics Committee ref: MSD-IDREC-C3-2014-010) and was performed according to the 1964 Declaration of Helsinki. Written informed consent was obtained from all participants prior to study inclusion.

\section{Design}

The study had a between-subject, double blind, placebo-controlled design. Participants 
were randomly assigned to a single dose of methylphenidate (Ritalin; 10mg oral dose, rapid release) or placebo (lactose-sucrose tablets), with the constraint that the groups were matched in size and gender distribution. In order to ensure that treatment was double blind, the methylphenidate and placebo tablets were of comparable weight and size and encapsulated in the same white vegetarian capsules (Dr. T \& T Health UK Ltd). Peak plasma concentration of methylphenidate is reached approximately 1-2 hours after administration and the half-life of the drug is estimated to be 2-4 hours (Gualtieri et al., 1982). Cognitive testing was therefore started 90 minutes after administration in order to maximise drug levels during the test session. All study visits began at 10am, with drug intake at approximately $11 \mathrm{am}$.

\section{Questionnaire measures}

Demographic information, handedness (Oldfield, 1971) and Verbal IQ (National Adult Reading Test, NART; Nelson and Willison, 1991) were measured at baseline. Questionnaires assessing mood and anxiety symptoms and perceived cognitive function were administered prior to treatment and after cognitive testing. These included the Beck Depression Inventory (BDI-II; Beck et al., 1996), Positive and Negative Affect Schedule (PANAS; Watson et al., 1988), State and Trait version of the State-Trait Anxiety Inventory (STAI-S and STAI-T; Spielberger et al., 1983)), the Perceived Deficits Questionnaire (PDQ; Sullivan et al., 1990)) and Visual Analogue Scales (VAS) 
assessing the following 16 items (Bond and Lader, 1974): Alert-Drowsy; Calm-Excited; Strong-Feeble; Fuzzy-Clear Headed; Well Coordinated-Clumsy; Lethargic-Energetic; Contented-Discontented; Troubled-Tranquil; Mentally Slow-Quick Witted; TenseRelaxed; Attentive-Dreamy; Incompetent-Proficient; Happy-Sad; AntagonisticFriendly; Interested-Bored; Withdrawn-Sociable. At the end of the study visit, participants were asked to guess which group they were in with a forced-choice question.

\section{Implicit Cognitive tests}

All participants were given the same tests in the same order, as described below.

Priming task

Priming is an implicit memory effect where prior exposure to a stimulus influences responses to the next stimulus. Negative priming tasks have previously been shown to engage inferior frontal and striatal (caudate nucleus) networks (Bauer et al 2012). In this task (based on Bauer et al., 2012), participants are presented with displays containing an "X” and an "O”, which can appear in two of four possible locations, and are required to indicate the position of the " $\mathrm{X}$ " while ignoring the "O". Each trial starts with the presentation of a fixation cross (jittered around $1500 \mathrm{~ms}$ ), followed by a prime display, to which participants have to respond. This is followed by a shorter fixation cross (400ms) and finally a probe display that participants have to respond to once again. 
Both prime and probe displays remain on the screen until a response has been made. In this task, there are two critical experimental conditions, and two control conditions, which are defined by the location of " $\mathrm{X}$ " and " $\mathrm{O}$ " in the probe display compared to the preceding prime display within the same trial (see Figure 1). The experimental conditions are 'repetition priming' in which the " $\mathrm{X}$ " is presented in the same location in the prime and probe trials and 'negative priming' in which probe display ' $\mathrm{X}$ ' is presented in the position previously occupied by the " $\mathrm{O}$ ” in the prime display. The two control conditions are: "control" in which both the probe display " $\mathrm{X}$ " and "O" are presented in locations unused in the prime display; and "imbalance" in which the probe " $\mathrm{X}$ " is presented in a previously unused location while the "O" appears where the " $\mathrm{X}$ " was presented in the prime display. The locations both " $\mathrm{X}$ " and " $\mathrm{O}$ " are presented in are thus balanced so that no single location is used more than the others. There are 48 trials per condition, resulting in a total of 192 trials. Only trials with correct responses to the prime are included in the analysis. Median reaction time values for each condition are calculated per participant. In order to assess priming effects, difference scores are calculated: Negative Priming (Negative - Control) and Repetition Priming (Control Repeat), calculating the time lost and gained as a result of negative and positive priming respectively in comparison to the control condition.

Contextual Cueing Task 
This task measures the implicit learning of contextual information and how it guides the deployment of visual attention. Neuropsychological studies have demonstrated that (extended) damage to the Middle Temporal Lobe (hippocampal) impairs performance on contextual cueing tasks (e.g. Chun and Phelps, 1999; Greene et al., 2007; Manns and Squire, 2001; Preston and Gabrieli, 2008). In this task (adapted from Chun and Jiang, 1998), the participant is required to report the orientation of a target (“T”), which is hidden amongst an array of distractor stimuli (“L”s). On half of the trials, the display array is a repeat of one previously presented and on the other half of trials the display array is novel. On each trial, a central fixation dot is presented for $500 \mathrm{~ms}$, followed by an stimulus array which includes the target stimulus "T" and 11 distractor stimuli (“L”s) that can appear in different orientations (rotations in all possible $90^{\circ}$ angles) in 48 prespecified locations. Participants are required to indicate the orientation of the target (which is rotated $90^{\circ}$ to the left or right) using a button press while keeping central fixation. The stimulus array is presented for 700ms (or until participant response). Reaction times greater than 2000ms are not recorded. There is a variable inter-trial interval (800ms, 1000ms, 1400ms). After a 2-minute practice block, the task runs for approximately 20 minutes (10 blocks of 48 trials). At the end of each block, the average reaction time and accuracy rate for that block are presented on the screen as feedback for the participant. EEG data was collected during this task (EEG data presented elsewhere). Median reaction time and accuracy are calculated per participant. In order to 
measure contextual learning, difference scores (novel vs. repeat) were computed for the first ( 5 blocks) and second (5 blocks) half of the task. Increased accuracy and decreased reaction times in repeated trials compared with novel trials are indicative of learning.

Implicit Intra-Dimensional-Extra-Dimensional Shift Task

The implicit intra-dimensional-extra-dimensional (IDED) shift task (adapted from Pringle et al., 2013) is a set-shift dot-probe task in which the location of a target stimulus is probabilistically predicted by a preceding stimulus (implicit rule learning) and the rules governing the relationship between the preceding stimulus and the target change over the course of the task (set-shifting). Set shifting tasks are known to engage fronto-striatal networks. The task is composed of 320 trials, broken up into four blocks (acquisition, reversal, extradimensional shift, and extradimensional reversal) of 80 trials each. In each trial, participants are presented with a central fixation cross for $750 \mathrm{~ms}$, which they are asked to fixate on. The fixation cross is then replaced with two shape stimuli, one at the top, and the other at the bottom of the screen, which are presented for 500ms. The stimuli vary in two dimensions (colour and shape) with the two stimuli presented in a trial differing on both. All the trials within a given block of the task use stimuli with the same two shapes which are presented in one of two colours (the specific stimuli used were drawn at random from a pool of six possible shapes and colours). 
Following the offset of the stimuli, a probe (either one or two small dots) is presented for $500 \mathrm{~ms}$ in the location of one of the stimuli. Participants are required to respond by button press to indicate the number of dots present in the probe. If participants have not responded within $1000 \mathrm{~ms}$ after the offset of the dot stimuli the trial is terminated. The task includes two critical manipulations: first, a specific exemplar of one of the stimulus dimensions (e.g. all circles, or all red stimuli) predicted the location of the probe in $80 \%$ of the trials with the other $20 \%$ of trials being surprising "catch trials"; second, the predictive exemplar changed between blocks. The first of these manipulations allows the degree to which participants learn to alter their attentional deployment to be measured. The logic behind this is that participants can use the predictive value of the stimuli to increase the efficiency of their responses; specifically participants should respond more accurately and quickly to probes in the predicted than the non-predicted location (this measure is effectively the "attentional vigilance" for the cued stimulus; MacLeod et al., 1986). In the current task, learning across a block may be inferred by comparing how much vigilance increases from the first to the last 40 trials in the block. The second manipulation allows assessment of whether participants are able to flexibly alter their attentional vigilance both within and across stimulus dimensions. In the initial (acq) block of the task, a particular stimulus colour, such as yellow, predicts probe location and therefore this block measures the ability of participants to learn the initial predictive properties of the stimulus. In the second block (rev), participants are 
presented with identical stimuli, however, the predictive stimulus changes to the other colour (e.g. if yellow predicted the probe in the first block, green predicts its location in the second). In the third block (ED acq), novel shapes and colours are used in the trials, and the predictive dimension alters so that shape is now predictive. In the final block (ED rev), a final reversal occurs so that the other shape now predicts probe location. For reaction time data, error trials were removed and median reaction time was calculated. Reaction time vigilance calculated as medianRT(catch_trials)-medianRT(predicitive_trials). Accuracy vigilance is calculated as accuracy(predicitive_trials)-accuracy(catch_trials). Both of these formulae result in a higher positive number if attention is directed towards the predictive stimuli.

\section{Explicit tasks}

Selected tasks were administered from the widely used CANTAB test battery (Cambridge Cognition, 2014), targeting reaction time measures, task switching abilities/cognitive flexibility, spatial working memory, association learning, and rapid visual processing. The Big Circle Little Circle task was included in order to familiarize participants with the CANTAB touch screen computer. In addition to assessing general reaction times of a participant, this task can be used to test task comprehension, learning and reversal learning. The Intra-dimensional-Extra-dimensional task (the explicit 
equivalent to the earlier described Implicit IDED, see implicit tasks) measures the ability to learn an underlying rule (rule acquisition), and the ability to learn new rules (reversal learning/set shifting). The Paired Association Learning task is a measure of episodic (visual) memory and new learning (association learning). The Spatial Working Memory task is a self-ordered search task that requires participants search through a spatial array of boxes on a screen to find hidden blue tokens. The Rapid Visual Processing task was used to assess sustained attention capacity.

Data analysis

All data were analysed using SPSS (IBM Corp. Released 2011. IBM SPSS Statistics for Windows, Version 22. Armonk, NY: IBM Corp). Baseline measures were compared between groups using independent samples t-tests for continuous data and chi-square tests for categorical data. Change in questionnaire measures over time was analysed using a split-plot analysis of variance (ANOVA) with the between subjects factors of treatment group and gender and the within subjects factor of time of assessment (pretreatment, post- treatment). Data from the cognitive tasks were analysed using split-plot ANOVAs with treatment group and gender as between-subjects factors and task conditions as within-subject factors. Where the assumption of sphericity was violated, degrees of freedom were corrected using Greenhouse-Geisser estimates of sphericity. 
Significant interactions were explored further using independent samples t-tests with Bonferroni correction.

\section{RESULTS}

Demographic data

There was no difference between the groups in age, Body Mass Index, verbal IQ, or handedness. The groups were well matched at baseline on scores of depression (Beck Depression Index), anxiety (Spielberger Trait Anxiety Index), affect (Positive and Negative Affect Scale), and perceived cognitive function (Perceived Deficits Questionnaire) (see Table 1). 


\begin{tabular}{|l|c|c|c|}
\hline & $\begin{array}{c}\text { Placebo } \\
(\mathrm{N}=40)\end{array}$ & $\begin{array}{c}\text { Methylphenidate } \\
(\mathrm{N}=40)\end{array}$ & Statistics \\
\hline Age: M(SD) & $22.5(3.0)$ & $23.3(3.1)$ & $\mathrm{p}=0.246$ \\
\hline Gender (Male:Female) & $20: 20$ & $20: 20$ & \\
\hline Verbal IQ: M(SD) & $118.7(6.4)$ & $117.4(6.1)$ & $\mathrm{p}=0.362$ \\
\hline Body Mass Index: M(SD) & $23.3(3.0)$ & $23.2(2.6)$ & $\mathrm{p}=0.825$ \\
\hline Handedness (Right: Left: Ambidextrous) & $32: 6: 2$ & $36: 2: 2$ & $\mathrm{p}=0.327$ \\
\hline Beck Depression Index: M(SD) & $3.2(3.8)$ & $4.5(4.6)$ & $\mathrm{p}=0.14$ \\
\hline Spielberger Trait Anxiety Index: M(SD) & $34.2(7.7)$ & $33.5(8.3)$ & $\mathrm{p}=0.72$ \\
\hline Positive and Negative Affect Scale: M(SD) & & & \\
\multicolumn{1}{|r|}{ Positive } & $29.3(7.0)$ & $30.8(6.8)$ & $\mathrm{p}=0.32$ \\
Negative & $12.1(2.8)$ & $12.2(2.2)$ & $\mathrm{p}=0.86$ \\
\hline Perceived Deficits Questionnaire: M(SD) & $18.7(7.8)$ & $18.7(10.6)$ & $\mathrm{p}=0.99$ \\
\hline
\end{tabular}

Table 1: Demographics and psychometrics of participants randomised to Methylphenidate $(n=40)$ or a matched placebo $(n=40)$. Verbal IQ was measured using the National Adult Reading Test (NART). Independent samples t-tests were conducted for all variables except handedness which was analysed using a Pearson chi-square test. Abbreviations: M mean, SD standard deviation.

Male participants were significantly heavier than female participants (mean (SD) weight: female 62.3kg (9.4); male 77.3kg (11.5), $\mathrm{t}(78)=6.4, \mathrm{p}<0.001)$. This difference resulted in a significant gender difference in the $\mathrm{mg} / \mathrm{kg}$ ratio of methylphenidate 
received by male and female participants (mean (SD) methylphenidate $\mathrm{mg} / \mathrm{kg}$ ratio: female methylphenidate group $0.166 \mathrm{mg} / \mathrm{kg}$ (0.027); male methylphenidate group 0.132 $\mathrm{mg} / \mathrm{kg}(0.018), \mathrm{t}(38)=4.7, \mathrm{p}<0.001)$. In order to control for this difference, participant weight was included in all subsequent analyses as a covariate.

There were no significant effects of methylphenidate on subjective measures of depression (Beck Depression Inventory), anxiety (Spielberger State-Trait Anxiety Index) or perceived cognitive function (Perceived Deficits Questionnaire), see Table 2.

\begin{tabular}{|c|c|c|c|c|c|}
\hline & \multicolumn{2}{|c|}{ Placebo $(\mathrm{N}=40)$} & \multicolumn{2}{|c|}{ Methylphenidate $(\mathrm{N}=40)$} & \multirow[t]{2}{*}{ Statistics } \\
\hline & $\begin{array}{c}\text { Pre- } \\
\text { treatment }\end{array}$ & $\begin{array}{c}\text { Post- } \\
\text { treatment }\end{array}$ & $\begin{array}{c}\text { Pre- } \\
\text { treatment }\end{array}$ & $\begin{array}{c}\text { Post- } \\
\text { treatment }\end{array}$ & \\
\hline Spielberger State Anxiety Index & $29.0(5.3)$ & $27.7(5.1)$ & $28.0(7.0)$ & $27.3(7.0)$ & $\mathrm{p}=0.55$ \\
\hline Spielberger Trait Anxiety & $34.2(7.7)$ & $32.6(7.4)$ & $33.5(8.3)$ & $31.9(7.4)$ & $\mathrm{p}=0.93$ \\
\hline Beck Depression Inventory & $3.2(3.8)$ & $2.8(3.7)$ & $4.6(4.6)$ & $3.5(3.7)$ & $\mathrm{p}=0.10$ \\
\hline Positive and Negative Affect Scale & & & & & \\
\hline Positive & $29.3(7.0)$ & $29.4(6.8)$ & $30.8(6.8)$ & $33.5(7.3)$ & $\mathrm{p}=0.02 *$ \\
\hline Negative & $12.1(2.8)$ & $11.6(2.1)$ & $12.2(2.2)$ & $12.2(3.1)$ & $p=0.21$ \\
\hline Perceived Deficits Questionnaire & $18.7(7.8)$ & $17.8(7.9)$ & $18.7(10.6)$ & $18.7(11.0)$ & $\mathrm{p}=0.25$ \\
\hline
\end{tabular}

Table 2: Subjective effects of acute methylphenidate and placebo on questionnaire measures of depression, anxiety, affect and cognitive function. Values represent the mean with standard deviation in parentheses. Each variable was analysed using a repeated measures ANOVA with group as a between subjects factor (placebo, methylphenidate) and time as a within groups factor (pre-treatment, post-treatment). The $\mathrm{p}$ values displayed are for the group $\mathrm{x}$ time interaction $(*=\mathrm{p}<0.05)$. 
However, there was a significant effect of acute methylphenidate on affect, as demonstrated by a three-way interaction between group (methylphenidate, placebo), time (pre-treatment, post-treatment) and valence (positive, negative) on PANAS scores $[F(1,76)=3.82, p=0.05]$. Separate analysis of the positive and negative scores, indicated that this interaction was driven by a significant interaction between group and time on the positive scores $[F(1,78)=5.65, p=0.02]$ but not the negative scores $[F(1,78)=1.67$, $\mathrm{p}=0.20]$. Post-hoc independent t-tests using the Bonferroni correction $(\mathrm{p}<0.025)$ clarified that PANAS positive scores were not different between the two groups at baseline $[\mathrm{t}(78)=1.01, \mathrm{p}=0.32]$, but that the methylphenidate group reported feeling significantly more positive post-treatment than the placebo group $[\mathrm{t}(78)=2.60, \mathrm{p}=0.01]$. The effect of methylphenidate on PANAS scores did not interact with gender.

The VAS scores were analysed with a repeated measures ANOVA with time (2 levels) and VAS item (16 levels) as within subject factors and drug group and gender as between subjects factors. There was a significant three-way time $\mathrm{x}$ item $\mathrm{x}$ drug group interaction $[F(15,1125)=, p=0.05]$. In order to understand this interaction further, the effect of drug group on each VAS item was investigated using separate repeated measures ANOVAs. There was a significant interaction between time and group for two of the items: Attentive-Dreamy $[\mathrm{F}(1,78)=5.79 ; \mathrm{p}=0.02]$ and Interested-Bored $[F(1,78)=10.38, p=0.002]$. In order to understand these interactions further, Bonferroniadjusted independent samples t-tests were performed to compare the ratings in each 
group at each time point $(\mathrm{p}<0.05 / 4=0.0125)$. There were no significant differences in ratings between the two groups at baseline. However, there was a significant difference between the two groups at the end of cognitive testing in ratings of Attentive-Dreamy $[\mathrm{t}(78)=3.78 ; \quad \mathrm{p}<0.001]$ and Interested-Bored $[\mathrm{t}(78)=3.30 ; \mathrm{p}=0.001]$, with the methylphenidate group reporting significantly higher levels of attentiveness and significantly lower levels of boredom. There were no significant interactions between time $\mathrm{x}$ group for any of the other VAS items.

In a forced-choice question about which group participants thought they were in (methylphenidate or Placebo), there was no significant difference in the responses between the two groups $[\mathrm{X}(1)=0.50 ; \mathrm{p}=0.48]$.

Priming Task

In order to assess the effect of acute methylphenidate on negative and repetition priming, the negative and repetition priming difference scores were entered into a repeated measures ANOVA with prime type (2 levels: negative, repetition) as the within-subjects factor and drug group and gender as between subjects factors. This revealed a significant three-way interaction between prime type $\mathrm{x}$ drug group $\mathrm{x}$ gender $[F(1,75)=3.96, p=0.05]$. In order to explore this interaction further, separate analyses of the male and female data were performed. In females, there was no significant interaction between prime type $\mathrm{x}$ group $[\mathrm{F}(1,37)=0.11, \mathrm{p}=0.74]$. In males there was a 
significant interaction between prime type $\mathrm{x}$ group $[\mathrm{F}(1,37)=7.82, \mathrm{p}=0.008]$, which was driven by an increase in positive priming in the methylphenidate group compared to placebo [t(38) $=2.72, \mathrm{p}=0.01$, Bonferroni threshold $\mathrm{P}<.025]$ but no effect of group on negative priming $[\mathrm{t}(38)=1.21, \mathrm{p}=0.24]$ (see Figure 1 ).

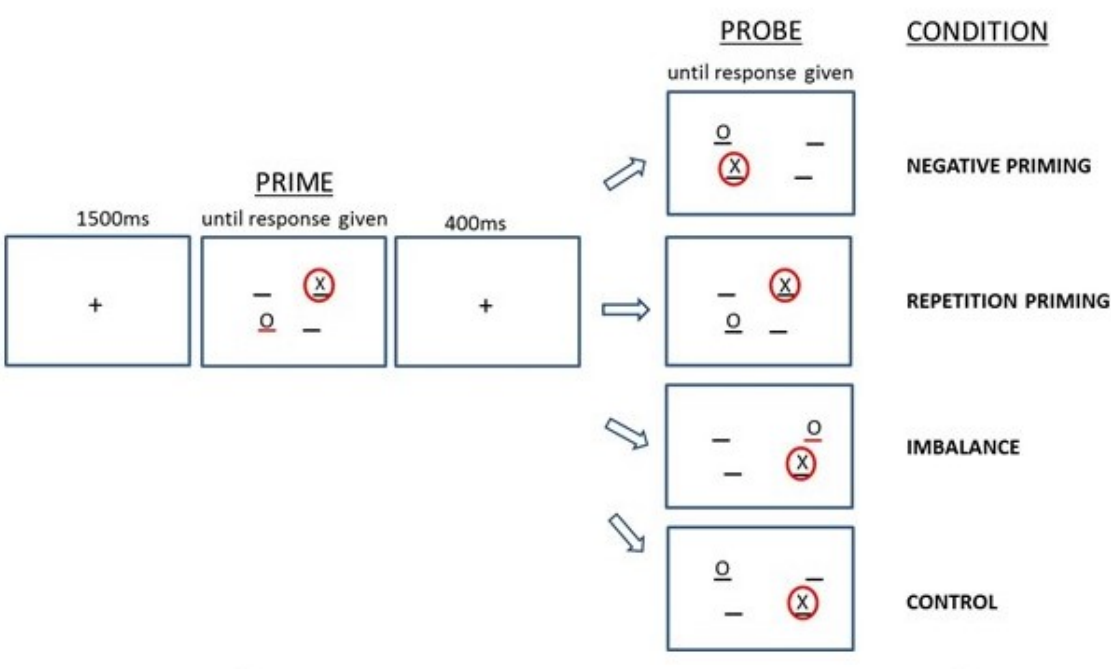

Females

Males

- Methylphenidate || Placebo

Methylphenidate $\approx$ Placebo
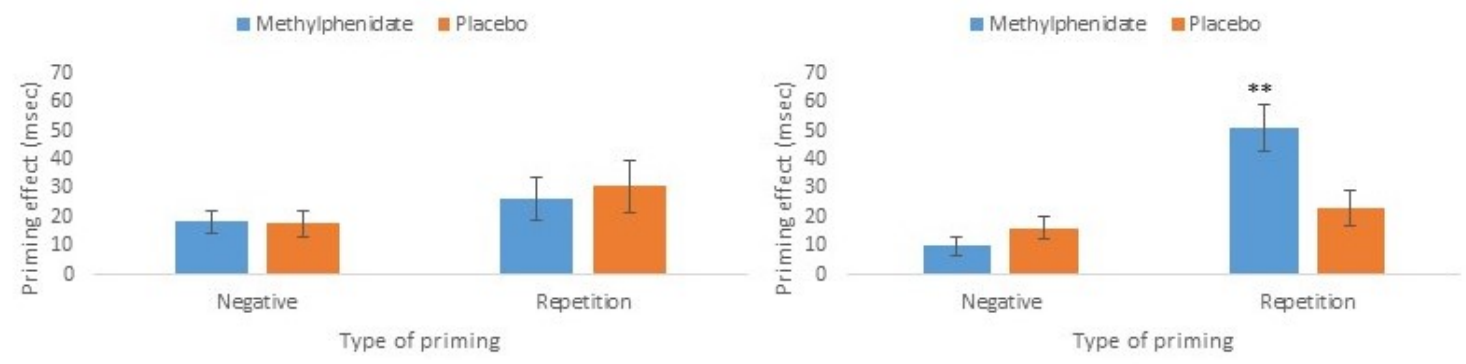

Figure 1 (upper): Examples of displays for the different trial types in the priming task. Red circles indicate the correct response. Figure 1 (lower): The effect of methylphenidate on positive and repetition priming in females (left) and males (right). 
The negative priming effect was calculated by subtracting reaction times in the control condition from reaction times in the negative priming condition. The repetition priming effect was calculated by subtracting reaction times in the repetition priming condition from reaction times in the control condition. ${ }^{* *}=p<0.01$.

There was no significant correlation between weight and positive priming effect in the methylphenidate group, suggesting that the significant difference in the effect of methylphenidate in male and female participants was not driven by weight differences.

Contextual cueing

In order to assess the effect of acute methylphenidate on contextual cueing, the accuracy difference scores (repeat-novel trials) were entered in to a repeated measures ANOVA with task stage (first 5 blocks vs last 5 blocks) as a within-subjects factor and betweensubjects factors of treatment group and gender. There was a significant effect of task stage [mean difference scores: first half $=2.125$, second half $=4.354 ; F(1,75)=5.49$, $\mathrm{p}=0.02]$ reflecting increased accuracy on the second half of the task compared with the first half on repeated trials compared with novel trials, which is consistent with contextual learning. However, this did not significantly interact with group. We also 
explored the effect of drug group on reaction time difference scores (novel-repeat trials), but there was no significant effect of task stage or drug group on this measure.

\section{Implicit IDED Task}

Data from the implicit IDED were analysed as learning rates (where learning rate is vigilance for the last half of the trials in the block-vigilance for the first half of the trials in the block) using repeated measures ANOVA with within-subjects factors of dimension (intra vs extradimensional; ID vs ED) and direction (acquisition vs reversal) and between-subjects factors of treatment group and gender. There was a significant main effect of dimension $[F(1,76)=4.324, p=0.041]$, as well as a significant three-way interaction between dimension, group and gender for accuracy learning rate $[F(1,76)=7.5, p=0.008]$.

When data from males and females was analysed separately, there was no significant effect of group on learning rates based on accuracy in the females (all p's $>0.1$ ). However in males, there was a significant interaction between dimension and drug group $[F(1,38)=5.58, p=0.02]$. Independent samples $t$-tests revealed that this interaction was driven by a relatively increased learning rate based on accuracy in the male methylphenidate group in the extradimensional condition compared with the male placebo group [t(38)=2.48, $\mathrm{p}=0.018$, Bonferonni-corrected threshold $\mathrm{p}<0.025]$. There was no significant difference between the groups in learning rate based on accuracy in 
the intradimensional condition $[\mathrm{t}(38)=0.78, \mathrm{p}=0.44]$. There was also a marginally significant three-way interaction between dimension, direction and group $[\mathrm{F}(1,76=3.28$, $\mathrm{p}=0.075$ ] for reaction time learning rate. In order to investigate this further, separate analyses were conducted for the intra- and extradimensional conditions, which indicated that the interaction was driven by a trend level interaction between direction and drug group $[\mathrm{F}(1,76)=2.94, \mathrm{p}=0.09]$ in the intra-dimensional condition. As can be seen in Figure 2, this interaction is driven by a relatively increased learning rate in the methylphenidate group during the acquisition phase compared to the placebo group. 
A $+$

B

Females

- Methylphenidate Placebo

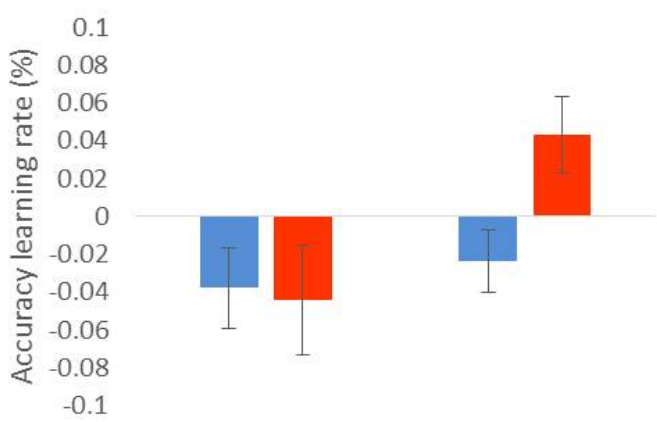

Intradimensional Extradimensional

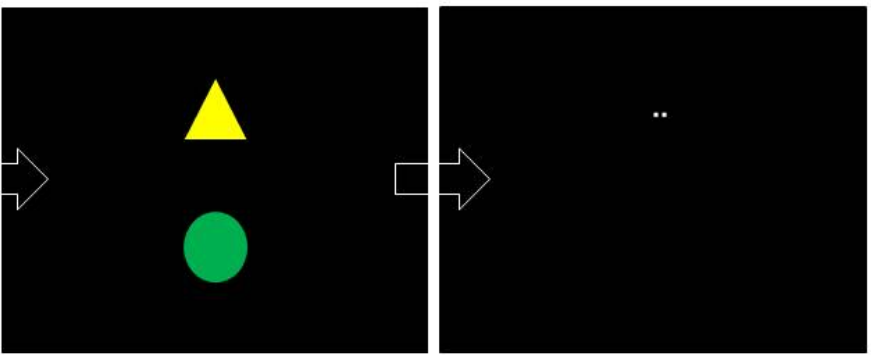

Males

- Methylphenidate $\quad$ Placebo

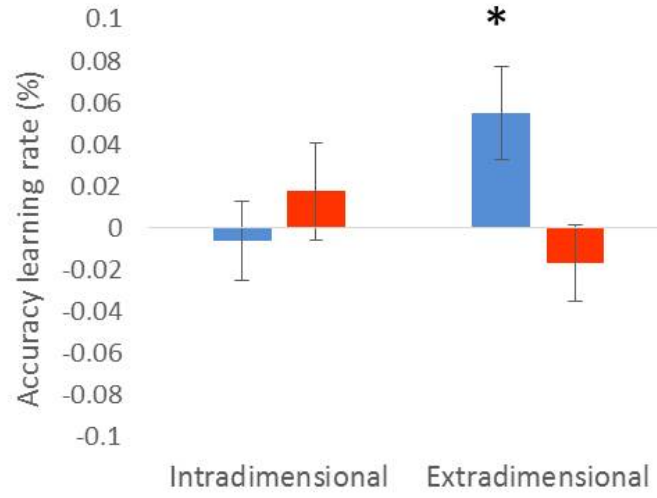

C

Females

Males

- Methylphenidate Placebo

- Methylphenidate Placebo
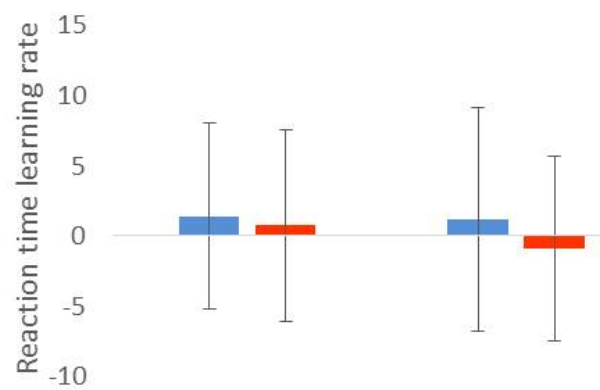

Intradimensional

Extradimensional

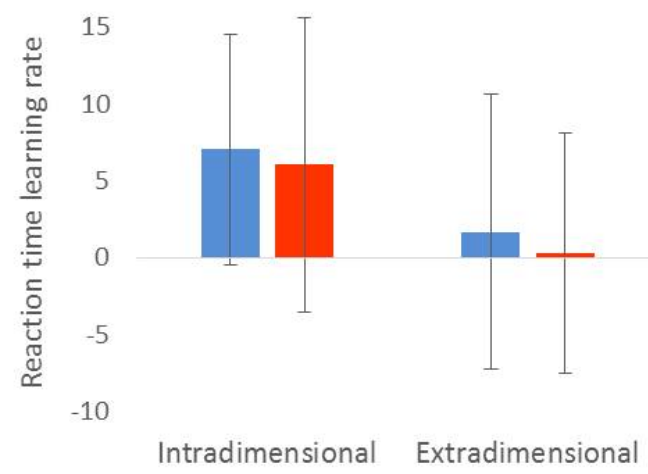


Figure 2 (A): Example trials from the implicit IDED task. In each trial, participants are presented with a central fixation cross that is replaced by two shapes. These are then followed by either one or two dots and the participant is required to indicate the number of dots, regardless of the location they appear in. Figure 2 (B): The effect of methylphenidate in females (left) and males (right) on learning rate, as measured by accuracy on the implicit IDED task. The learning rate was calculated by subtracting accuracy vigilance in the first half of the block from vigilance in the last half of the block. Figure $2(C)$ : The effect of methylphenidate in females (left) and males (right) on learning rate, as measured by reaction times on the implicit IDED task. The learning rate was calculated by subtracting reaction time vigilance in the second half of the block from vigilance in the first half of the block. * $p<0.05$

In the extra-dimensional condition, there was no significant main effect of group $[F(1,76)=0.05, p=0.83]$ or interaction between drug and direction $[F(1,76)=0.88$, $\mathrm{p}=0.35]$.

Explicit tasks

There was no significant effect of drug group, or interaction between drug group and 
gender, on accuracy or reaction times on the Big Circle Little Circle Task (all p’s>0.4).

Likewise, there was no significant effect of group or interaction between group, gender or task stage on number of errors on the Explicit IDED Task (all p's >0.4).

On the Paired Association Learning Task, there was also no main effect of group or interaction between group, gender and condition (2,3,6 and 8 shapes) on number of errors or number of trials to reach criterion (all p's $>0.6$ ).

On the Spatial Working Memory Task, there was no main effect of group or interaction between group, gender and condition $(4,6,8,10$ boxes) on total errors or mean time to last response (all p’s>0.1). However, there was a significant interaction between condition, group and gender on the strategy measure $[F(3,228)=3.48, p=0.03]$. Separate analyses revealed no significant effect of group or group $\mathrm{x}$ condition interaction in female participants (all p’s>0.5). However, in males, there was a significant interaction between group and condition $[F(3,114)=5.36, p=0.002]$. Bonferroni corrected independent samples t-tests for each condition $(\mathrm{p}<0.05 / 4)$ revealed significantly higher strategy scores (reflecting worse strategy) in the 8 box $[\mathrm{t}(38)=2.79, \mathrm{p}=0.008]$ and 10 box $[\mathrm{t}(38)=3.08, \mathrm{p}=0.004]$ conditions in the male methylphenidate group compared with the male placebo group. 
There was no difference between the two groups on the Rapid Visual Processing Task in terms of probability of hit (identifying the target sequence), total hits, total misses and mean latency (all p's $>0.1$ ).

\section{DISCUSSION}

A low dose of methylphenidate enhanced implicit learning in both the priming task and the implicit IDED task in this sample of healthy volunteers. There was no evidence for improved learning in any of the explicit measures including the explicit version of the IDED task. Indeed, methylphenidate appeared to increase explicit strategy levels in the spatial working memory task, which is reflective of poorer performance. The results are consistent with our hypothesis that implicit measures of cognition are more sensitive to pharmacological-induced enhancement in healthy volunteer models than the traditionally employed, more explicit measures.

Methylphenidate increased the repetition priming effect in healthy male participants compared with placebo. Thus, male participants in the methylphenidate group showed a greater enhancement of their performance of the task following a repetition of the target stimulus in the same location compared to a neutral control location. Methylphenidate 
also improved performance on the implicit intra-dimensional extradimensional task, indexed by changes in accuracy vigilance learning rate measures. Compared to placebo, methylphenidate enhanced accuracy learning rates in the extradimensional blocks in male participants, which is consistent with the notion that methylphenidate improves implicit rule learning in healthy male participants. This effect underscores our previous report of an effect of a single dose of modafinil on this task (Pringle et al., 2013) and suggests that the implicit IDED task is sensitive to the effects of a range of cognition enhancing drugs with different neurochemical profiles in healthy volunteers. It is important to note that the effect of modafinil on this task was seen on learning rate as indexed by reaction time, whereas the effect of methylphenidate seen in the current study was on learning rate as indexed by accuracy. There was also a suggestion that methylphenidate also increased reaction time learning rates across both male and female participants; however, this should be interpreted with caution given the trend-level significance.

In line with our hypothesis, there were no beneficial effects of methylphenidate on explicit cognition. Indeed, there were unexpectedly increased strategy scores in the methylphenidate group on the spatial working memory task, which is suggestive of poorer performance. Whilst it is challenging to interpret an effect on the strategy measure in the absence of other markers of poorer performance (such as reduced accuracy or increased reaction times) such a finding is clearly in contrast to a number of 
previous studies that have reported improved spatial working memory following methylphenidate in both adult patients with ADHD (Agay et al., 2014; Turner et al., 2005) and healthy volunteers (Agay et al., 2014; Cooper et al., 2005; Elliott et al., 1997; Mehta et al., 2000). It is important to note that these previous studies all used a higher dose of methylphenidate, which may explain the discrepancy in findings. However, other studies that have used a range of doses of methylphenidate $(10 \mathrm{mg}, 20 \mathrm{mg}, 40 \mathrm{mg}$ and 60mg) have reported no difference on measures of spatial working memory, suggesting that dosage cannot fully account for the discrepant findings in the literature (Clatworthy et al., 2009; Linssen et al., 2012).

Interestingly, the effects of methylphenidate on implicit cognitive performance in the current study were observed in males, but not in females. This is consistent with evidence from a recent meta-analysis (Ilieva et al., 2015), which demonstrated larger effects of methylphenidate on cognition in studies with a larger proportion of healthy male participants. It is important to note, that there was a significant difference in weight between the male and female groups in the current study, which meant that, on average, the female group received a higher $\mathrm{mg} / \mathrm{kg}$ dose than the males. This raises the possibility that the greater effect of methylphenidate on cognition in males was driven by the lower dose of the drug received by this group. However, in order to control for this, participant weight was included as a covariate in all analyses and the effects of sex persisted. The sex differences seen in the current study are consistent with the known 
influence of sex on the dopamine (DA) system. For example, there are well-established sex differences in the incidence, prevalence, clinical course and treatment outcome of a range of disorders that are associated with DA dysfunction, including schizophrenia, Parkinson's Disease and ADHD (Abel et al., 2010; Biederman et al., 2002; Crawford and DeLisi, 2016; Dahodwala et al., 2016; Davies, 2014; Keezer et al., 2016; Mendrek and Mancini-Marïe, 2016). Further, animal models and human postmortem and neuroimaging studies have highlighted a strong influence of sex on DA neurotransmission and striatal and extrastriatal DA D2 receptor density (Castner and Becker, 1996; Di Paolo, 1994; Kaasinen et al., 2001; Konradi et al., 1992; Laakso et al., 2002; Munro et al., 2006; Riccardi et al., 2011).

The clear effects of methylphenidate on subjective mood ratings support the idea that an acute $10 \mathrm{mg}$ dose was sufficient to exert psychoactive effects. PET studies suggest that such a dose would have been expected to produce approximately $40 \%$ blockade of dopamine transporter 120 minutes after administration (Volkow et al., 1998). Consistent with these predicted neurochemical effects, participants in the methylphenidate group reported feeling significantly more positive and alert at the end of the test session and significantly less bored. Such effects are in line with the subjective effects reported in previous healthy volunteer studies that employed a higher dose of methylphenidate (e.g. (Elliott et al., 1997). 
However, the $10 \mathrm{mg}$ acute dose of methylphenidate used in the current study was lower than the dose used in many previous studies of the effects of methylphenidate in healthy volunteers and the lack of findings on the explicit measures used should be interpreted in light of this. This dose was chosen following a recent meta-analysis suggesting that this may be an optimum dosage to see effects on the cognitive domains targeted in this study (Linssen et al., 2014) and to minimize side effects in the healthy volunteers. A low dose was also chosen in light of the hypothesized inverted U-shaped relationship between cognitive performance and DA activity (Cools and D'Esposito, 2011; Goldman-Rakic et al., 2000; Robbins and Arnsten, 2009; Robbins and Sahakian, 1979). Animal and human studies have illustrated that both low and excessively high levels of DA impair working memory and reversal learning performance. Whilst in conditions, such as ADHD, methylphenidate is thought to act by restoring deficient levels of DA, healthy volunteers are thought to have near optimum levels of catecholamines. Therefore increasing DA and noradrenaline (NA) levels through the pharmacological blockade of the DA transporter might lead, in some situations, to a decrement in performance. In support of this, there is evidence that those with poorer baseline working memory capacity show larger positive effects of methylphenidate on cognitive performance (Agay et al., 2014; del Campo et al., 2013; Mehta et al., 2000; van der Schaaf et al., 2013). Given the relatively high functioning participants included in the current study, a low dose of methylphenidate was chosen to try to limit the possibility of 
such negative effects of the drug on cognitive performance.

Despite the increasingly widespread usage of cognition enhancing drugs, such as methylphenidate, by healthy individuals seeking to enhance concentration and academic performance (Dance, 2016; Franke et al., 2013; Singh et al., 2014; Smith and Farah, 2011), the evidence that these pharmacological agents increase cognitive function in healthy volunteers has been challenging to establish (Advokat, 2010; Hall and Lucke, 2010; Ilieva et al., 2015; Smith and Farah, 2011). Although some studies report positive effects of methylphenidate on cognition (Agay et al., 2014; Clatworthy et al., 2009; del Campo et al., 2013; Elliott et al., 1997; Mehta et al., 2000), these effects are highly variable (Linssen et al., 2014; van der Schaaf et al., 2013). While effects are observed overall in meta-analyses, these are difficult to detect and characterize in individual studies. One explanation for this variability is the poor sensitivity of the measures of cognition to subtle changes in performance in healthy volunteers (Husain and Mehta, 2011). Often the standard cognitive test batteries used are designed to test cognitive change in clinical groups who have already experienced some loss of function. Such measures typically lack dynamic range when used in healthy volunteers and can be affected by ceiling effects or the use of compensatory strategies. Indeed, previous studies in healthy volunteers have demonstrated that the effect of methylphenidate on cognition in healthy volunteers is only seen on tasks that are sufficiently demanding (Vaidya et al., 1998). Previous studies support the hypothesis that implicit measures of 
cognition may be a more sensitive marker both of pharmacological enhancement (Pringle et al., 2013) and progression of illness in disorders such as Parkinson's disease (Filoteo et al., 2007). Here we show that the neuro-enhancing effects of methylphenidate are also more readily detected on implicit tasks, even at a relatively low dose, in high functioning healthy participants. As such, implicit measures of cognition may be a useful way of screening and tracking cognitive effects of novel agents in experimental medicine studies and in early phase studies in patient groups as they depend on striatal involvement that has been shown to be especially sensitive to factors such as aging (e.g. Raz et al., 2005), multiple diseases (e.g. in Parkinson's Disease; Foerde and Shohamy, 2011; van Asselen et al., 2009), as well as pharmacological challenges (e.g. Cools et al., 2001). Future studies are needed to elucidate the effects of methylphenidate and other cognitive enhancers on these implicit cognitive measures in clinical populations and to test whether such effects are predictive of the clinical effects of the drug. 
AUTHOR CONTRIBUTIONS: PM and CJH conceived the study. CK, CS, ACN and CJH developed the task design. Testing and data collection were performed by CK and CS. Data analysis was conducted by CK and SEM, and the interpretation of the data was performed by CK, SEM and CJH. The first draft of the manuscript was written by SEM, CK and CJH. Revisions were provided by all authors. All authors approved the final version of the manuscript for submission.

ACKNOWLEDGEMENTS: This research was funded by a grant from UCB through the UCB-Oxford Alliance and supported by the NIHR Oxford Health Biomedical Research Centre. The views expressed are those of the authors and not necessarily those of the NHS, the NIHR or the Department of Health. The authors thank NE Myers for invaluable input regarding the Contextual Cueing task.

DECLARATION OF CONFLICTING INTERESTS: CK and CS declare no competing interests. PM is full time employee of UCB pharma, a pharmaceutical company who commercialize and develop drugs for CNS disorders. SEM has received consultancy payments from p1Vital and has participated in paid speaking engagements for Lilly UK. CJH has received consultancy fees from P1vital and Lundbeck and has received grant income from UCB, J\&J, Sunovion and Lundbeck. 


\section{REFERENCES}

Abel KM, Drake R and Goldstein JM (2010) Sex differences in schizophrenia. International Review of Psychiatry (Abingdon, England) 22(5): 417-428.

Advokat C (2010) What are the cognitive effects of stimulant medications? Emphasis on adults with attention-deficit/hyperactivity disorder (ADHD). Neuroscience and Biobehavioral Reviews 34(8): 1256-1266.

Agay N, Yechiam E, Carmel Z, et al. (2014) Methylphenidate enhances cognitive performance in adults with poor baseline capacities regardless of attention-deficit/hyperactivity disorder diagnosis. Journal of Clinical Psychopharmacology 34(2): 261-265.

Bauer E, Gebhardt H, Gruppe H, et al. (2012a) Altered negative priming in older subjects: first evidence from behavioral and neural level. Frontiers in Human Neuroscience 6. Available from: http://www.ncbi.nlm.nih.gov/pmc/articles/PMC3461575/ (accessed 9 January 2015).

Bauer E, Gebhardt H, Gruppe H, et al. (2012b) Altered negative priming in older subjects: first evidence from behavioral and neural level. Frontiers in Human Neuroscience 6. Available from: http://journal.frontiersin.org/article/10.3389/fnhum.2012.00270/abstract (accessed 28 February 2017).

Beck AT, Steer RA and Brown GK (1996) Manual for the Beck Depression Inventory-II. In: San Antonio: TX: Psychological Corporation. Available from: http://www.nctsn.org/content/beck-depression-inventory-second-edition-bdi-ii (accessed 28 February 2017).

Biederman J, Mick E, Faraone SV, et al. (2002) Influence of gender on attention deficit hyperactivity disorder in children referred to a psychiatric clinic. The American Journal of Psychiatry 159(1): 36-42.

Bond $A$ and Lader $M(1974)$ The use of analogue scales in rating subjective feelings. British Journal of Medical Psychology 47(3): 211-218.

Cambridge Cognition (2014) CANTAB $^{\circledR}$ [Cognitive assessment software]. Cambridge Cognition. Available from: www.cantab.com.

Castner SA and Becker JB (1996) Sex differences in the effect of amphetamine on immediate early gene expression in the rat dorsal striatum. Brain Research 712(2): 245-257. 
Chun MM and Jiang Y (1998) Contextual cueing: implicit learning and memory of visual context guides spatial attention. Cognitive Psychology 36(1): 28-71.

Chun MM and Phelps EA (1999) Memory deficits for implicit contextual information in amnesic subjects with hippocampal damage. Nature Neuroscience 2(9): 844-847.

Clatworthy PL, Lewis SJG, Brichard L, et al. (2009) Dopamine release in dissociable striatal subregions predicts the different effects of oral methylphenidate on reversal learning and spatial working memory. The Journal of Neuroscience: The Official Journal of the Society for Neuroscience 29(15): 4690-4696.

Cools R and D'Esposito M (2011) Inverted-U-shaped dopamine actions on human working memory and cognitive control. Biological Psychiatry 69(12): e113-125.

Cools R, Barker RA, Sahakian BJ, et al. (2001) Enhanced or Impaired Cognitive Function in Parkinson's Disease as a Function of Dopaminergic Medication and Task Demands. Cerebral Cortex 11(12): 1136-1143.

Cooper NJ, Keage H, Hermens D, et al. (2005) The dose-dependent effect of methylphenidate on performance, cognition and psychophysiology. Journal of Integrative Neuroscience 4(1): 123-144.

Crawford MB and DeLisi LE (2016) Issues related to sex differences in antipsychotic treatment. Current Opinion in Psychiatry 29(3): 211-217.

Dahodwala N, Pei Q and Schmidt P (2016) Sex Differences in the Clinical Progression of Parkinson's Disease. Journal of Obstetric, Gynecologic \& Neonatal Nursing 45(5): 749756.

Dance A (2016) Smart drugs: A dose of intelligence. Nature 531(7592): S2-S3.

Davies W (2014) Sex differences in attention Deficit Hyperactivity Disorder: candidate genetic and endocrine mechanisms. Frontiers in Neuroendocrinology 35(3): 331-346.

del Campo N, Fryer TD, Hong YT, et al. (2013) A positron emission tomography study of nigrostriatal dopaminergic mechanisms underlying attention: implications for ADHD and its treatment. Brain: A Journal of Neurology 136(Pt 11): 3252-3270.

Di Paolo T (1994) Modulation of brain dopamine transmission by sex steroids. Reviews in the Neurosciences 5(1): 27-41. 
Elliott R, Sahakian BJ, Matthews K, et al. (1997) Effects of methylphenidate on spatial working memory and planning in healthy young adults. Psychopharmacology 131(2): 196-206.

Filoteo JV, Maddox WT, Salmon DP, et al. (2007) Implicit category learning performance predicts rate of cognitive decline in nondemented patients with Parkinson's disease. Neuropsychology 21(2): 183-192.

First M, Spitzer R, Gibbon M, et al. (2002) Structured Clinical Interview for DSM-IV-TR Axis I Disorders, Research Version, Non-patient Edition. (SCID-I/NP). New York: Biometrics Research: New York State Psychiatric Institute.

Foerde K and Shohamy D (2011) The role of the basal ganglia in learning and memory: insight from Parkinson's disease. Neurobiology of Learning and Memory 96(4): 624-636.

Franke AG, Bagusat C, Dietz P, et al. (2013) Use of illicit and prescription drugs for cognitive or mood enhancement among surgeons. BMC medicine 11: 102.

Goldman-Rakic PS, Muly EC and Williams GV (2000) D(1) receptors in prefrontal cells and circuits. Brain Research. Brain Research Reviews 31(2-3): 295-301.

Greene AJ, Gross WL, Elsinger CL, et al. (2007) Hippocampal differentiation without recognition: An fMRI analysis of the contextual cueing task. Learning \& Memory 14(8): 548-553.

Gualtieri CT, Wargin W, Kanoy R, et al. (1982) Clinical studies of methylphenidate serum levels in children and adults. Journal of the American Academy of Child Psychiatry 21(1): 1926.

Hall WD and Lucke JC (2010) The enhancement use of neuropharmaceuticals: more scepticism and caution needed. Addiction (Abingdon, England) 105(12): 2041-2043.

Harmer CJ, Cowen PJ and Goodwin GM (2011) Efficacy markers in depression. Journal of Psychopharmacology (Oxford, England) 25(9): 1148-1158.

Husain M and Mehta MA (2011) Cognitive enhancement by drugs in health and disease. Trends in Cognitive Sciences 15(1): 28-36.

Ilieva IP, Hook CJ and Farah MJ (2015) Prescription Stimulants' Effects on Healthy Inhibitory Control, Working Memory, and Episodic Memory: A Meta-analysis. Journal of Cognitive Neuroscience 27(6): 1069-1089. 
Kaasinen V, Någren K, Hietala J, et al. (2001) Sex differences in extrastriatal dopamine d(2)-like receptors in the human brain. The American Journal of Psychiatry 158(2): 308-311.

Keezer MR, Wolfson C and Postuma RB (2016) Age, Gender, Comorbidity, and the MDS-UPDRS: Results from a Population-Based Study. Neuroepidemiology 46(3): 222-227.

Konradi C, Kornhuber J, Sofic E, et al. (1992) Variations of monoamines and their metabolites in the human brain putamen. Brain Research 579(2): 285-290.

Laakso A, Vilkman H, Bergman J, et al. (2002) Sex differences in striatal presynaptic dopamine synthesis capacity in healthy subjects. Biological Psychiatry 52(7): 759-763.

Linssen AMW, Vuurman EFPM, Sambeth A, et al. (2012) Methylphenidate produces selective enhancement of declarative memory consolidation in healthy volunteers. Psychopharmacology 221(4): 611-619.

Linssen AMW, Sambeth A, Vuurman EFPM, et al. (2014) Cognitive effects of methylphenidate in healthy volunteers: a review of single dose studies. The International Journal of Neuropsychopharmacology 17(6): 961-977.

MacLeod C, Mathews A and Tata P (1986) Attentional bias in emotional disorders. Journal of Abnormal Psychology 95(1): 15-20.

Manns JR and Squire LR (2001) Perceptual learning, awareness, and the hippocampus. Hippocampus 11(6): 776-782.

Mehta MA, Owen AM, Sahakian BJ, et al. (2000) Methylphenidate enhances working memory by modulating discrete frontal and parietal lobe regions in the human brain. The Journal of Neuroscience: The Official Journal of the Society for Neuroscience 20(6): RC65.

Mendrek A and Mancini-Marïe A (2016) Sex/gender differences in the brain and cognition in schizophrenia. Neuroscience and Biobehavioral Reviews 67: 57-78.

Millan MJ, Agid Y, Brüne M, et al. (2012) Cognitive dysfunction in psychiatric disorders: characteristics, causes and the quest for improved therapy. Nature Reviews. Drug Discovery 11(2): 141-168.

Munro CA, McCaul ME, Wong DF, et al. (2006) Sex differences in striatal dopamine release in healthy adults. Biological Psychiatry 59(10): 966-974. 
NHS Prescription Cost Analysis England 2004 and 2015 (n.d.) NHS Digital. Available from: http://content.digital.nhs.uk/.

Oldfield, RC (1971) The assessment and analysis of handedness: the Edinburgh inventory. 1(9): 97-113.

Preston AR and Gabrieli JDE (2008) Dissociation between Explicit Memory and Configural Memory in the Human Medial Temporal Lobe. Cerebral Cortex 18(9): 2192-2207.

Pringle A, Browning M, Parsons E, et al. (2013) Early markers of cognitive enhancement: developing an implicit measure of cognitive performance. Psychopharmacology 230(4): 631-638.

Raz N, Lindenberger U, Rodrigue KM, et al. (2005) Regional Brain Changes in Aging Healthy Adults: General Trends, Individual Differences and Modifiers. Cerebral Cortex 15(11): 1676-1689.

Repantis D, Schlattmann P, Laisney O, et al. (2010) Modafinil and methylphenidate for neuroenhancement in healthy individuals: A systematic review. Pharmacological Research 62(3): 187-206.

Riccardi P, Park S, Anderson S, et al. (2011) Sex Differences in the relationship of regional Dopamine release to affect and cognitive function in Striatal and Extrastriatal Regions using PET and [18F]Fallypride. Synapse (New York, N.Y.) 65(2): 99-102.

Robbins TW and Arnsten AFT (2009) The neuropsychopharmacology of fronto-executive function: monoaminergic modulation. Annual Review of Neuroscience 32: 267-287.

Robbins TW and Sahakian BJ (1979) 'Paradoxical' effects of psychomotor stimulant drugs in hyperactive children from the standpoint of behavioural pharmacology.

Neuropharmacology 18(12): 931-950.

Rogers RD, Blackshaw AJ, Middleton HC, et al. (1999) Tryptophan depletion impairs stimulusreward learning while methylphenidate disrupts attentional control in healthy young adults: implications for the monoaminergic basis of impulsive behaviour.

Psychopharmacology 146(4): 482-491.

Singh I, Bard I and Jackson J (2014) Robust Resilience and Substantial Interest: A Survey of Pharmacological Cognitive Enhancement among University Students in the UK and Ireland. PLOS ONE 9(10): e105969. 
Smith ME and Farah MJ (2011) Are prescription stimulants 'smart pills'? The epidemiology and cognitive neuroscience of prescription stimulant use by normal healthy individuals. Psychological Bulletin 137(5): 717-741.

Spielberger C, Gorsuch R, Lushene R, et al. (1983) Manual for the State-Trait Anxiety Inventory. Palo Alto, CA: Consulting Psychologists Press.

Sullivan MJ, Edgley K and Dehoux E (1990) A survey of multiple sclerosis: I. Perceived cognitive problems and compensatory strategy use. Canadian Journal of Rehabilitation 4(2): 99105.

Turner DC, Blackwell AD, Dowson JH, et al. (2005) Neurocognitive effects of methylphenidate in adult attention-deficit/hyperactivity disorder. Psychopharmacology 178(2-3): 286295.

Vaidya CJ, Austin G, Kirkorian G, et al. (1998) Selective effects of methylphenidate in attention deficit hyperactivity disorder: a functional magnetic resonance study. Proceedings of the National Academy of Sciences of the United States of America 95(24): 1449414499.

van Asselen M, Almeida I, Andre R, et al. (2009) The role of the basal ganglia in implicit contextual learning: A study of Parkinson's disease. Neuropsychologia 47(5): 12691273.

van der Schaaf ME, Fallon SJ, Ter Huurne N, et al. (2013) Working memory capacity predicts effects of methylphenidate on reversal learning. Neuropsychopharmacology: Official Publication of the American College of Neuropsychopharmacology 38(10): 2011-2018.

Volkow ND, Wang GJ, Fowler JS, et al. (1998) Dopamine transporter occupancies in the human brain induced by therapeutic doses of oral methylphenidate. The American Journal of Psychiatry 155(10): 1325-1331.

Watson D, Clark LA and Tellegen A (1988) Development and validation of brief measures of positive and negative affect: the PANAS scales. Journal of Personality and Social Psychology 54(6): 1063-1070. 